\title{
1 Mathematical modeling reveals a complex network of signaling and 2 apoptosis pathways in the survival of memory plasma cells
}

\section{Philipp Burt ${ }^{1,2}$, Rebecca Cornelis ${ }^{1}$, Gustav Geißler ${ }^{1,2}$, Stefanie Hahne ${ }^{1}$, Andreas Radbruch ${ }^{1}$, 4 Hyun-Dong Chang ${ }^{1,3, *}$, Kevin Thurley ${ }^{1,2,4, *}$}

$5 \quad{ }^{1}$ German Rheumatism Research Center, Berlin, Germany

$6 \quad{ }^{2}$ Institute for Theoretical Biology, Humboldt University, Berlin, Germany

$7 \quad{ }^{3}$ Department of Cytometry, Institute of Biotechnology, Technical University, Berlin, Germany

$8{ }^{4}$ Biomathematics, Institute for Experimental Oncology, University Hospital Bonn, Bonn, Germany

9 * Correspondence:

10 chang@drfz.de

11 kevin.thurley@uni-bonn.de

\section{Abstract}

13 The long-term survival of memory plasma cells is conditional on the signals provided by dedicated 14 survival niches in the bone marrow organized by mesenchymal stromal cells. Recently, we could show 15 that plasma cell survival requires secreted factors such as APRIL and direct contact to stromal cells, 16 which act in concert to activate NF-kB- and PI3K-dependent signaling pathways to prevent cell death. 17 However, the precise dynamics of the underlying regulatory network are confounded by the complexity 18 of potential interaction and cross-regulation pathways. Here, based on flow-cytometric quantification

19 of key signaling proteins in the presence or absence of the required survival signals, we generated a 20 quantitative model of plasma cell survival. Our model emphasizes the non-redundant and essential 21 nature of the two plasma cell survival signals APRIL and stromal cell contact, providing resilience to 22 endoplasmic reticulum stress and mitochondrial stress, respectively. Importantly, the modeling 23 approach allowed us to unify distinct data sets and derive a consistent picture of the intertwined 24 signaling and apoptosis pathways regulating plasma cell survival. 


\section{Introduction}

The vertebrate immune system has the unique ability to provide long-term protection against pathogens it has encountered previously. An important cellular correlate of this long-term immunity is the longlived memory plasma cell which constitutively secretes copious amounts of specific antibodies. Antibodies produced by memory plasma cells serve as a highly efficient barrier against re-infection with pathogens, but have also been associated with a variety of autoimmune diseases (1-3). Memory plasma cell survival is conditional on signals provided to them in dedicated survival niches, organized by mesenchymal stromal cells most prominently in the bone marrow, where memory plasma cells can survive for decades $(1,3-6)$. The survival of memory plasma cells is mediated through the integration of multiple input signals, including integrin-mediated contact to mesenchymal stromal cells and signaling via B cell maturation antigen (BCMA) and Transmembrane activator and CAML interactor (TACI) (7,8). Recently, we demonstrated that provision of A proliferation inducing ligand (APRIL), addressing BMCA, and cell contact to a stromal cell, maintains primary bone marrow memory plasma cells in vitro via NF-kB (nuclear factor 'kappa-light-chain-enhancer' of activated B-cells) and PI3K (phosphatidylinositol 3-kinase) signaling pathways, respectively (9). We could show that APRIL and cell contact address different cellular stress pathways, namely the endoplasmic reticulum stress and mitochondrial stress, respectively. However, the architecture of the intracellular signaling network regulating the resilience to these stressors and thereby promoting plasma cell survival remains poorly understood.

A prominent regulatory mechanism restricting the life-span of plasma cells is the widely studied Bcl2-associated X protein (BAX)-dependent apoptosis pathway (10). Apoptosis is regulated by extrinsic and intrinsic signals and ultimately results in the activation of caspases, a family of cysteine proteases. Mitochondria play a fundamental role in the coordination of the apoptotic pathways. Oligomerization of two proteins, BAX and BAK (BCL2-antagonist/killer 1), that are localized at the mitochondrial outer membrane and cytosol, lead to the formation of the apoptotic pore. Pore formation precedes cytochrome $\mathrm{c}$ and apoptosis-inducing factor release into the cytosol and downstream activation of caspases. The BCL-2 family comprises a group of proteins that is critical for the control of apoptosis by regulating the oligomerization of BAX and BAK. Localized mainly at the mitochondria, they can

53 be divided into pro- and anti-apoptotic proteins. The anti-apoptotic proteins (B-cell lymphoma 2, BCL2

54 and myeloid cell leukemia 1, MCL1) prevent apoptotic pore formation and preserve the integrity of 55 the mitochondrial membrane by binding to BAX and BAK. Pro-apoptotic proteins (Bcl-2-like protein 
11, BIM and NOXA), next to BAX and BAK, compete in binding to the anti-apoptotic proteins thereby neutralizing them. Thus, the ratio of pro- to anti-apoptotic proteins plays a decisive role in apoptosis regulation (11). The expression of the anti-apoptotic proteins BCL2 and MCL1 was shown to be upregulated in plasma cells, but while BCL2 seems to be dispensable for the maintenance of memory plasma cells, MCL1 is essential for their survival (12). In multiple myeloma, MCL1 strongly binds to BIM thereby blocking apoptosis (13). In addition to BIM, MCL1 was reported to interact with NOXA (14).

The complexity of the apoptotic network and the design principles leading to the apoptosis decision have been investigated extensively both by experiment and mathematical models. Pioneered by the work of Fussenegger and colleagues, mechanistic differential-equation based models of caspase signaling were introduced to study apoptosis systems $(15,16)$. Subsequent studies focusing on caspase activation in single cells showed that this process is rapid and irreversible $(17,18)$. Many experimental and computational studies have since then successfully demonstrated that the apoptosis decision is well-described by a bi-stable and reversible switch (19-21). Several combined efforts of modeling and experiment have focused on quantitative aspects of the apoptotic network either in large-scale studies or focusing on individual control points of the apoptosis network (22,23). While many of the mechanisms regulating apoptosis have been quantified and modeled, it is less clear how different antiapoptotic extracellular input signals affect the delicate machinery of cell death, and how well cell-type specific aspects are captured by the available modeling framework.

In this work, we developed a mathematical model of plasma cell survival in the bone marrow, using published $(9,24)$ and yet unpublished data on key components of the plasma cell apoptosis pathway. To this end, we started from a core model based on established principles of the BAX-dependent apoptosis pathway, by adding the extracellular input signals APRIL and ST2 cells, and subsequently, by considering a more detailed interaction network of several caspase proteins downstream of the BAX-module. We found that the survival factors APRIL and ST2 cells have differential rather than additive roles in the regulation of plasma-cell lifespan extension, acting in different ways and primarily on different parts of the network. Further, our analysis underlines the essential aspect of differential caspase regulation for the apoptosis decision and provides insight into the parameters that could be manipulated to alter plasma cell lifespan. 


\section{Methods}

\section{Cell culture and Flow cytometric measurements of apoptosis proteins}

88 Experiments were performed with the same cell culture system as previously reported (9). Intracellular

89 antigens were stained by fixing cells with PFA and permeabilization with methanol. To prevent 90 unspecific binding, cells were pre-incubated with blocking buffer and subsequently stained with 91 primary antibody for 1 hour and, if necessary, with secondary antibody for 30 minutes. Samples were 92 analyzed using a MacsQuant analyzer and FlowJo software. Cytometric procedures followed the 93 recommendations of the "Guidelines for use of flow cytometry and cell sorting in immunological 94 studies" (25). The following antibodies were used in the experiments:

\begin{tabular}{|l|l|l|}
\hline Anti-mouse BCL2, REA356 & Miltenyi Biotec & Catalog \# 130-105-474 \\
\hline Anti-mouse BIM, 14A8 & Milipore & Catalog \# MAB17001 \\
\hline Anti-mouse CD138, REA104 & Miltenyi Biotec & Catalog \# 130-102-318 \\
\hline Anti-mouse MCL1, Y37 & Abcam & Catalog \# ab32087 \\
\hline Anti-mouse NOXA, 114C307 & Abcam & Catalog \# ab13654 \\
\hline
\end{tabular}

\section{Data analysis and statistics}

961 To estimate the half-life for plasma cells under different conditions, we fitted an exponential decay

97 function $\boldsymbol{f}(\boldsymbol{t})=\mathbf{1 0 0} \boldsymbol{e}^{-\lambda t}$ to each individual time series. The resulting decay rates were converted to

98 half-lives for each condition according to: $\boldsymbol{t}_{\mathbf{1} / \mathbf{2}}=\log (2) / \lambda$, where $\lambda$ represents the average decay rate 99 summarized from the individual fit procedures. To compare average protein concentrations, geometric 100 means for each protein measured under a specific condition were first normalized to the respective 101 medium condition and then compared using an unpaired Student's t-test. Uncertainties of protein ratios 102 were calculated from the normalized protein concentrations by bootstrapping. To this end, we 103 repeatedly drew from the original samples with replacement to estimate mean and standard deviation 104 of the data.

\section{Mathematical models and numerical simulations}

106 All model simulations were carried out in Python 3.8. Ordinary differential equations were solved 107 using the scipy.odeint routine. For curve-fitting, least-squares optimization was employed using the 108 Levenberg-Marquardt algorithm implemented in the Python lmfit library. For the perturbation 109 analysis, we defined the effect size as the $\log 2$ fold-change of half-life (or BAX*) between a model 
110 simulation with best-fit parameter values and the model simulation with perturbed parameters. The

111 parameters were either up- or downregulated by one order of magnitude.

112 a) BAX-dependent apoptosis: We consider (i) production and degradation of MCL-2 family proteins

113 in dependence of the input stimuli APRIL and ST2, (ii) complex formation between pro- and anti-

114 apoptotic proteins (see Figure 2B), and (iii) a dependence of the average half-life of the plasma cell

115 population on the average concentration of activated $\mathrm{BAX}\left(\mathrm{BAX}^{*}\right)$ as follows:

$$
\lambda=\gamma \frac{\left[B A X^{*}\right]^{3}}{K_{B a x}^{3}+\left[B A X^{*}\right]^{3}} .
$$

116 A complete description of the model equations is available as Supplemental Text, parameters are listed 117 in Table 1.

118 b) BAX-independent regulation of caspases: To consider the effect of direct caspase regulation by 119 APRIL and ST2 on the apoptosis decision, we extended the BAX-Apoptosis model and assumed 120 additional regulation of the cell-death rate $\lambda$ as follows (see Figure $3 \mathrm{~A}$ and text):

$$
\lambda=\left[\gamma\left(\frac{[B A X]^{3}}{K_{B a x}^{3}+[B A X]^{3}}+\frac{\kappa}{1+\alpha f([A P R I L])}\right)\left(\frac{1}{1+\beta f([S T 2])}\right)\right] .
$$

121 That means, the cell-death rate $\gamma$ is increased due to the activity of caspase $3 / 7$, which is induced 122 through a combination of BAX-dependent and independent effects. Those BAX-independent effects 123 stem from activity of caspase 12, which can be inhibited by APRIL. Finally, the activity of caspase 3/7

124 can be inhibited by ST2. Here, the fitting parameter $\kappa$ is the relative effect of caspase 12 activity, the 125 fitting parameters $\beta$ denotes the maximal inhibitory effect ST2 on caspase 3/7, and we set $\alpha=10$ for 126 the inhibitory effect size of APRIL. For model fitting to our data in absence and presence of APRIL

127 and ST2, we adopt a Boolean formulation for the regulatory function, $f(x)=\left\{\begin{array}{ll}1, & x>0 \\ 0, & x=0\end{array}\right.$. Modification 128 of this function into a more specific Hill-type form such as $f(x)=x^{n} /\left(x^{n}+K\right)$ is straight-forward.

\section{Results}

\section{A quantitative model of BAX-dependent apoptosis in plasma cells}

131 Previous experiments have shown that plasma cells are not intrinsically long-lived but that provision 132 of the cytokine APRIL combined with cell-cell contact, conferred by the stromal cell line ST2, keeps 133 them alive in vitro, while each factor alone proved to be insufficient (9)(Figure 1A). Indeed, re-plotting 
134 the data of (8) separately for individual experiments revealed an exponential survival curve for cultured

135 plasma cells, where the half-life is significantly increased from less than 1 day to more than 6 days

136 after addition of APRIL and ST2 (Figure 1B-C and Figure S1). How these signals integrate and affect

137 the underlying complex regulatory network facilitating apoptosis decisions remains unclear (Figure

138 1A). In particular, we wondered whether the experimental observations could be reconciled with an

139 additive model, where APRIL and ST2 synergistically act to inhibit BAX-dependent apoptosis, or

140 alternatively, whether APRIL and ST2 address fundamentally distinct parts of the apoptosis network.

141 Since in plasma cells, APRIL stimulates the NF-kB signaling pathway (26-28)(8) and ST2 acts via the

142 PI3K pathway to stimulate the transcription factor FoxO $(9,29)$, we started our analysis based on the

143 working-hypothesis that ST2 and APRIL act by targeting components of the BAX-dependent apoptosis

144 pathway. To derive a specific mathematical model, we measured the abundance of several key

145 components of the BCL-2 family, namely BIM, BCL-2, NOXA and MCL-1, after 3 days in our

146 established in vitro culture system (9) with and without stimulation by APRIL and ST2 by

147 immunofluorescent single-cell staining (Figure 2A). We found that the presence of APRIL alone had

148 a tendency to affect BCL-2 ( $\mathrm{p}=0.123)$, whereas ST2 had a significant negative effect on BIM, NOXA

149 and MCL-1 concentrations (Figure 2A). The divergent effects of APRIL and ST-2 on different

150 members of the BCL-2 family were even more pronounced when considering the ratios within pairs of

151 pro- and anti-apoptotic proteins (Figure 2B). We used that information to establish a refined regulatory

152 network of BAX-dependent plasma-cell apoptosis (Figure 2C). Combined with a recently published

153 compilation of quantitative data for the BCL-2 interactome (Table 1)(24,30-33), we were now in a

154 position to formulate and annotate a specific mathematical model of BAX-dependent apoptosis in

155 plasma cells (Methods and Supplementary Text). The only parameters lacking good estimates from the

156 literature are the values describing protein production rates, which we therefore used as fitting

157 parameters. Indeed, the resulting model was able to describe the new data set of BCL-2 family member

158 protein abundance (Figure 2D).

159 Having established a mathematical formulation of the BAX-dependent apoptosis pathway in the 160 context of plasma cell survival, we proceeded to the original question of whether APRIL and ST2-

161 dependent regulation of that pathway are sufficient to explain the observed survival kinetics shown in

162 Figure 1B. Interestingly, we found that our model was well able to fit the protein and survival data

163 individually (Figure S2), but it was not able to capture both protein and survival data at the same time

164 within the expected range of the experimental data (Figure 2E). In fact, the annotated model predicted

165 a positive regulatory effect of ST2 on the average level of activated BAX (BAX*) in the plasma cell 
166 population (Figure 2F). That ST2-dependent elevation of BAX* cannot be fully compensated for by

167 APRIL, which explains the disconnect of that data-derived model parameterization with our cell-

168 survival data (Figure 2E). Hence, we concluded that APRIL and ST2-driven regulation of BAX-

169 dependent apoptosis alone is insufficient to explain the observed survival curves, and rather, additional

170 regulatory mechanisms must be considered.

171 Direct regulation of Caspase proteins is required for effective control

172 Caspases are not only mediators, but also critical regulators of apoptosis $(34,35)$, and our recent data 173 suggest that APRIL and ST2 have different roles in their regulation (9). Specifically, we found that

174 ST2 inhibits caspases 3 and 7, whereas APRIL inhibits the ER-stress-induced apoptosis mediated via 175 caspase 12. Therefore, we supplemented our core model of BAX-dependent apoptosis by considering 176 a network of caspase regulation (Figure 3A)(Methods). To specify the model, we added the 2 new 177 fitting parameters describing the extended caspase network, and we found that this extended model 178 indeed captures the individual and combined effects of APRIL and ST2 (Figure 3B-C). In particular, 179 in contrast to the BAX core model, we obtained good results fitting the new caspase-related parameters 180 to the plasma cell survival data (Figure 3C).

181 The successful data annotation gave us the opportunity to analyze the dependency on individual stimuli 182 and on different parts of the pathway in more detail. Interestingly, we found that in the model, 183 overexpression of anti-apoptotic proteins is far more effective than knock-down of pro-apoptotic 184 proteins in the regulation of activated BAX (Figure 3D). Further, regulation of caspase 3/7 turned out 185 to have the largest effect on plasma cell life-span (Figure 3E). That is in good agreement with the 186 intuition gained from Figure 2E, that direct regulation of caspase 3/7 through ST2 is required to 187 counteract the pro-apoptotic role of ST2 within the BAX-dependent apoptosis pathway. Taken 188 together, our analysis suggests that a unified description of the two considered data sets requires 189 consideration of both BAX-dependent and BAX-independent regulation of caspases via both APRIL 190 and ST2.

\section{Full model topology is essential to describe plasma cell survival}

192 To further test whether our proposed model topology was necessary to describe all available data, we 193 derived a set of sub-models lacking one or more components of the full model (Figure 4A). For each 194 possible combination, we fitted the model to the available data and compared the fitting result to the 195 original model fit (Figure 4B and Figure S3). The full model had clearly the smallest fitting error $\left(\chi^{2}\right)$, 
and models lacking regulatory effects of either APRIL, ST2 or both on caspase activity all provided similar fitting quality (Figure 4B). For a refined model comparison, we employed Akaike's information criterion (AIC) (Figure 4C), a well-established metric for comparing models with differing numbers of fitting parameters (36). AIC values lack a direct interpretation, and thus we only consider differences between models (- $\triangle \mathrm{AIC})$, here we show $-\triangle \mathrm{AIC}$ in relation to the model with poorest fit quality (Figure $4 C)$. A difference of $\triangle \mathrm{AIC}>2$ is usually regarded as significant, and therefore, our analysis clearly rules out all sub-models (Figure $4 \mathrm{C}$ ). Nevertheless, the $\triangle \mathrm{AIC}$ representation can also be regarded as a ranking of the most critical model components for explanation of the available data. As such, it is intriguing that in our model, ST2-driven regulation of caspase 3/7 is the most critical individual factor (panel (iv) in Figure 4A and C), even exceeding the effect of by-passing caspase regulation completely (panel (vi)). Hence, our model analysis supports both a strong role for BAX-independent caspase regulation and the critical need of ST2-derived signals for effective regulation of plasma cell survival.

\section{Discussion}

In this work, we developed a mathematical model describing the survival of plasma cells in the bone marrow. We used the model to unify two acquired data sets, namely abundance of pro- and antiapoptotic proteins and plasma-cell survival kinetics in the presence of the soluble factor APRIL and/or stromal ST2 cells as cell-contact dependent survival signal. In the annotated model, the balance of survival proteins is a critical factor for the lifespan of plasma cells. Further, we found that a combination of direct caspase regulation together with regulation of BAX-dependent pathways is essential to explain all available data.

A special feature in plasma cell biology is the contribution of the endoplasmic reticulum (ER) to apoptosis regulation $(10,37)$. Since plasma cells as the primary antibody-secreting cell type produce vast amounts of proteins, they experience high ER-stress due to protein misfolding. In plasma cells, BAX was not only found in the mitochondrial membrane but also localized at the ER (10). We could recently show that the plasma cell microenvironment, composed of stromal cell contact and the cytokine APRIL, counteracts the activation of caspases 3 and 7 and caspase 12, respectively. Caspases 3 and 7, that are activated upon mitochondrial stress (34), are regulated by PI3K signaling that is activated by cell contact to stromal cells. Activation of the ER-associated caspase 12 is inhibited by APRIL signaling via the NF-kB pathway (9). Here, our model simulations supported the view that apoptosis. 
In vivo, memory plasma cells persist for a lifetime, i.e. they do not have a dedicated half-life: data indicate that plasma cells survive as long as they are provided with their niche (38-40). Our in vitro culturing system cannot yet reflect such very long life-times, which we think is mainly due to technical limitations - the co-cultured ST2 cells start overgrowing the plasma cells after $\sim 5$ days in culture. Also, we cannot exclude additional regulatory factors accounting for the very long life-times reported in vivo. However, the observed time-scale separation between a half-life of $\sim 1$ day for medium condition and of 6 days in presence of APRIL and ST2 rather argues against the same apoptosis mechanism

234 driving the fast and the slow plasma cell decline. Therefore, in model development, we considered the APRIL+ST2 condition as a base-line reflecting properties of the cell culturing system distinct from apoptosis regulation in the context of long-lived plasma cells.

237 The life-span of immune cell populations is tightly controlled and is a critical property in a range of inflammatory processes, as shown previously in the context of selective expansion of lymphocyte subtypes $(41,42)$ and NK cell subpopulations $(43)$, amongst others. For therapy, targeted depletion of memory plasma cells has been proposed as a promising strategy in autoimmune diseases such as lupus erythematosus (2). On the other hand, memory plasma cells can provide long-lasting protection against infections, as recently demonstrated for mild SARS-Cov2 infections in humans (44). In plasma cell biology, quantitative studies have previously focused on the generation and composition of the survival niche (45) and on the long-term development of the pool of available memory plasma cells (46), but our quantitative understanding of apoptosis signaling pathways in plasma cells is still quite limited. Here, using cultured plasma cells as a model system, we developed a mathematical modeling framework to study apoptosis regulation in specific immune cell populations in the context of established signaling pathways.

\section{Funding}

251 This work was supported by the Leibniz association (Junior Research Group program, to K.T.), the 252 Leibniz ScienceCampus Chronic inflammation, the Deutsche Forschungsgemeinschaft (TH 1861/4-1, 253 to K.T. and SFB TRR130 P16 to H.D.C. and A.R.) and the Innovative Medicines Initiative 2 Joint 254 Undertaking under grant agreement no. 777357 to H.D.C. and A.R. H.D.C. is supported by the Dr. 255 Rolf M. Schwiete Foundation. 
bioRxiv preprint doi: https://doi.org/10.1101/2021.08.26.457784; this version posted August 28, 2021. The copyright holder for this preprint (which was not certified by peer review) is the author/funder, who has granted bioRxiv a license to display the preprint in perpetuity. It is made available under aCC-BY-NC-ND 4.0 International license.

\section{Supplementary Material}

258 The supplementary material contains 3 Figures and the detailed description and equations of the BAX-

259 dependent apoptosis model. 


\section{References}

262 1. Khodadadi L, Cheng Q, Radbruch A, Hiepe F. The Maintenance of Memory Plasma Cells. Front Immunol (2019) 10: doi:10.3389/fimmu.2019.00721

264 2. Hiepe F, Radbruch A. Plasma cells as an innovative target in autoimmune disease with renal manifestations. Nat Rev Nephrol (2016) 12:232-240. doi:10.1038/nrneph.2016.20

3. Maschmeyer P, Chang H-D, Cheng Q, Mashreghi M-F, Hiepe F, Alexander T, Radbruch A. Immunological memory in rheumatic inflammation - a roadblock to tolerance induction. Nat

4. Nutt SL, Hodgkin PD, Tarlinton DM, Corcoran LM. The generation of antibody-secreting plasma cells. Nat Rev Immunol (2015) 15:160-171. doi:10.1038/nri3795

271 5. Chu VT, Berek C. The establishment of the plasma cell survival niche in the bone marrow. Immunol Rev (2013) 251:177-188. doi:10.1111/imr.12011

6. Manz RA, Thiel A, Radbruch A. Lifetime of plasma cells in the bone marrow. Nature (1997) 388:133-134. doi:10.1038/40540 Memory B Cell Depletion during CD20 Immunotherapy in Mice. J Immunol (2008) 180:361371. doi:10.4049/jimmunol.180.1.361 Immunol (2008) 180:3655-3659. doi:10.4049/jimmunol.180.6.3655

9. Cornelis R, Hahne S, Taddeo A, Petkau G, Malko D, Durek P, Thiem M, Heiberger L, Peter L,

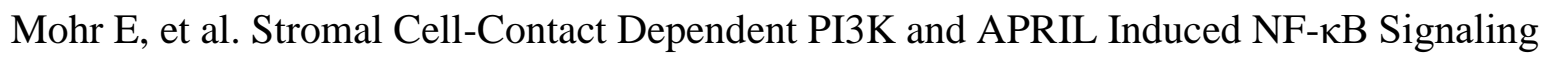
Prevent Mitochondrial- and ER Stress Induced Death of Memory Plasma Cells. Cell Rep (2020) 32: doi:10.1016/j.celrep.2020.107982 
11. Cosentino K, García-Sáez AJ. Bax and Bak Pores: Are We Closing the Circle? Trends Cell Biol (2017) 27:266-275. doi:10.1016/j.tcb.2016.11.004

12. Peperzak V, Vikström I, Walker J, Glaser SP, Lepage M, Coquery CM, Erickson LD, Fairfax K, MacKay F, Strasser A, et al. Mcl-1 is essential for the survival of plasma cells. Nat Immunol (2013) 14:290-297. doi:10.1038/ni.2527

13. Gomez-Bougie P, Bataille R, Amiot M. The imbalance between Bim and Mcl-1 expression controls the survival of human myeloma cells. Eur J Immunol (2004) 34:3156-3164. doi:10.1002/eji.200424981

14. Nakajima W, Hicks MA, Tanaka N, Krystal GW, Harada H. Noxa determines localization and

15. Fussenegger M, Bailey JE, Varner J. A mathematical model of caspase function in apoptosis. Nat Biotechnol (2000) 18:768-774. doi:10.1038/77589

16. Spencer SL, Sorger PK. Measuring and modeling apoptosis in single cells. Cell (2011) 144:926-939. doi:10.1016/j.cell.2011.03.002

17. Goldstein JC, Waterhouse NJ, Juin P, Evan GI, Green DR. The coordinate release of (2000) 2:156-162. doi:10.1038/35004029

18. Rehm M, Düßmann H, Jänicke RU, Tavaré JM, Kögel D, Prehn JHM. Single-cell fluorescence resonance energy transfer analysis demonstrates that caspase activation during apoptosis is a rapid process: Role of caspase-3. J Biol Chem (2002) 277:24506-24514.

311 19. Eissing T, Conzelmann H, Gilles ED, Allgöwer F, Bullinger E, Scheurich P. Bistability analyses of a caspase activation model for receptor-induced apoptosis. J Biol Chem (2004)

314 20. Legewie S, Blüthgen N, Herzel H. Mathematical modeling identifies inhibitors of apoptosis as 
mediators of positive feedback and bistability. PLoS Comput Biol (2006) 2:1061-1073. doi:10.1371/journal.pcbi.0020120

21. Chen C, Cui J, Lu H, Wang R, Zhang S, Shen P. Modeling of the role of a Bax-activation switch in the mitochondrial apoptosis decision. Biophys $J$ (2007) 92:4304-4315.

22. Janes KA, Gaudet S, Albeck JG, Nielsen UB, Lauffenburger DA, Sorger PK. The response of human epithelial cells to TNF involves an inducible autocrine cascade. Cell (2006) 124:1225-

23. McKenna S, García-Gutiérrez L, Matallanas D, Fey D. BAX and SMAC regulate bistable properties of the apoptotic caspase system. Sci Rep (2021) 11:1-15. doi:10.1038/s41598-021-

24. Hantusch A, Brunner T, Frickey T, Rehm M. Bcl-2-Ome-a database and interactive web service for dissecting the Bcl-2 interactome. Cell Death Differ (2017) 24:192.

25. Cossarizza A, Chang HD, Radbruch A, Acs A, Adam D, Adam-Klages S, Agace WW, doi:10.1038/cdd.2016.129

26. Hatzoglou A, Roussel J, Bourgeade M-F, Rogier E, Madry C, Inoue J, Devergne O, Tsapis A. Aghaeepour N, Akdis M, Allez M, et al. Guidelines for the use of flow cytometry and cell sorting in immunological studies (second edition). Eur J Immunol (2019) 49:1457-1973. doi:10.1002/eji.201970107

27. Chu VT, Fröhlich A, Steinhauser G, Scheel T, Roch T, Fillatreau S, Lee JJ, Löhning M, Berek TNF Receptor Family Member BCMA (B Cell Maturation) Associates with TNF ReceptorAssociated Factor (TRAF) 1, TRAF2, and TRAF3 and Activates NF- $\kappa$ B, Elk-1, c-Jun N- 
29. Van Spriel AB, De Keijzer S, Van Der Schaaf A, Gartlan KH, Sofi M, Light A, Linssen PC, orchestrates the $\alpha 4 \beta 1$ integrin-Akt signaling axis and supports long-lived plasma cell survival.

30. Rooswinkel RW, Van De Kooij B, De Vries E, Paauwe M, Braster R, Verheij M, Borst J. Antiapoptotic potency of Bcl-2 proteins primarily relies on their stability, not binding selectivity. Blood (2014) 123:2806-2815. doi:10.1182/blood-2013-08-519470

31. Cao X, Deng X, May WS. Cleavage of Bax to p18 Bax accelerates stress-induced apoptosis, and a cathepsin-like protease may rapidly degrade p18 Bax. Blood (2003) 102:2605-2614.

32. Meller R, Cameron JA, Torrey DJ, Clayton CE, Ordonez AN, Henshall DC, Minami M, doi:10.1182/blood-2003-01-0211

33. Dengler MA, Weilbacher A, Gutekunst M, Staiger AM, Vöhringer MC, Horn H, Ott G, pathway mediates short-term ischemic tolerance in cultured neurons. J Biol Chem (2006)

34. Lakhani SA, Masud A, Kuida K, Porter GA, Booth CJ, Mehal WZ, Inayat I, Flavell RA. Aulitzky WE, van der Kuip H. Discrepant NOXA (PMAIP1) transcript and NOXA protein endoplasmic-reticulum-specific apoptosis and cytotoxicity by amyloid- $\beta$. Nature (2000)

36. Cavanaugh JE, Neath AA. The Akaike information criterion: Background, derivation, properties, application, interpretation, and refinements. Wiley Interdiscip Rev Comput Stat (2019) 11:e1460. doi:10.1002/wics.1460 
37. Gass JN, Gunn KE, Sriburi R, Brewer JW. Stressed-out B cells? Plasma-cell differentiation and the unfolded protein response. Trends Immunol (2004) 25:17-24.

38. Landsverk OJB, Snir O, Casado RB, Richter L, Mold JE, Réu P, Horneland R, Paulsen V, doi:10.1016/j.it.2003.11.004

39. Zehentmeier S, Roth K, Cseresnyes Z, Sercan Ö, Horn K, Niesner RA, Chang HD, Radbruch

40. Hoyer BF, Moser K, Hauser AE, Peddinghaus A, Voigt C, Eilat D, Radbruch A, Hiepe F, Manz RA. Short-lived plasmablasts and long-lived plasma cells contribute to chronic humoral

41. Gett A V, Hodgkin PD. A cellular calculus for signal integration by T cells. Nat Immunol (2000) 1:239-244.

42. Heinzel S, Binh Giang T, Kan A, Marchingo JM, Lye BK, Corcoran LM, Hodgkin PD. A

43. Hammer Q, Rückert T, Borst EM, Dunst J, Haubner A, Durek P, Heinrich F, Gasparoni G, Myc-dependent division timer complements a cell-death timer to regulate $\mathrm{T}$ cell and $\mathrm{B}$ cell responses. Nat Immunol (2017) 18:96-103. doi:10.1038/ni.3598

44. Turner JS, Kim W, Kalaidina E, Goss CW, Rauseo AM, Schmitz AJ, Hansen L, Haile A, Klebert MK, Pusic I, et al. SARS-CoV-2 infection induces long-lived bone marrow plasma cells in humans. Nature (2021)1-8. doi:10.1038/s41586-021-03647-4

45. Mohr M, Hose D, Seckinger A, Marciniak-Czochra A. Quantification of plasma cell dynamics using mathematical modeling. R Soc Open Sci (2018) 5: doi:10.1098/rsos.170759 
bioRxiv preprint doi: https://doi.org/10.1101/2021.08.26.457784; this version posted August 28,2021 . The copyright holder for this preprint (which was not certified by peer review) is the author/funder, who has granted bioRxiv a license to display the preprint in perpetuity. It is made available under aCC-BY-NC-ND 4.0 International license.

EO, Dörner T, et al. Adaptation of humoral memory. Immunol Rev (2006) 211:295-302.

399 doi:10.1111/j.0105-2896.2006.00380.x 
Figure 1: Regulation of plasma cell survival via APRIL and ST2. (A) Schematic signal integration of ST2 and APRIL. How the signals are integrated into the Bax-dependent apoptosis network is yet unclear. Time course were data taken from Ref. (9) and are here represented as Box plots. Data were fitted to exponential curves, each experiment at a time (Figure S1). Curves shown here represent mean+s.e.m. of those fit results. C) Average half-lives of each condition based on the fitting procedure from $(\mathrm{B}) .{ }^{*} * \mathrm{P}<0.01, * * * \mathrm{P}<0.001, \mathrm{n}>12$ fits per condition. Error bars represent standard deviation.

(A) Geometric mean of measured core proteins in APRIL/ST2/APRIL+ST2 environments normalized to the respective concentration without stimulus (Medium). (B) Ratios of indicated proteins taken from

411 (A) after normalization to Medium condition. (C) Model scheme based on the effects of APRIL and 412 ST2 on MCL-2 family protein abundance data shown in A. The model includes pro-apoptotic proteins 413 BIM and NOXA (red) and anti-apoptotic proteins BCL-2 and MCL-1 (green). (D) Protein ratios (see 414 A) fitted to the model shown in panel B. Error bars represent standard deviation. (E) Model fit to 415 survival kinetics (Figure 1B) after fitting the protein ratios. (F) Time-course simulation of the 416 mechanistic apoptosis model for different inputs as indicated.

417 Figure 3: Unifying the data sets on MCL-2 family members and plasma cell survival data 418 requires direct regulation of caspases. (A) Model scheme, combination of the mechanistic 419 mitochondrial apoptosis model (see Figure 2B) with ER-stress-induced caspase activation. (B-C) 420 Combined model fit to protein data and survival kinetics. Error bars represent standard deviation. (D421 E) Effect on half-life and BAX activation for simulated knockdown or overexpression of proteins in 422 the apoptosis network. Growth rates for protein species were varied by one order of magnitude in both 423 directions.

424 Figure 4: All considered regulatory processes are required to explain the data. (A) Different 425 hypothetic network topologies (ii-vi) as submodules of the model shown in (3A), here presented as a 426 condensed version (i). Abbreviations: M2: Model 2; A: APRIL; S:ST2; C12: Caspase 12; C3/7: 427 Caspase 3, Caspase 7. Model components removed from the full model are shown in red. (B) goodness428 of-fit (1/root-mean-squared error)) for each submodel (ii-vi). (C) Akaike information criterion (- $\triangle \mathrm{AIC})$ 429 for each sub-model compared to the full model (i), where the model with smallest $-\Delta$ AIC is used as a 430 reference and set to 0 . 
431 Table 1: Parameter values used in the mathematical models.

\begin{tabular}{|c|c|c|c|c|}
\hline Parameter & Value & Unit & Role & Source \\
\hline $\mathrm{a}_{\mathrm{BCL}-2}$ & 0.11 & - & Effect APRIL on $g_{\text {BCL-2 }}$ & Fit \\
\hline SMCL-1 & 0.37 & - & Effect ST2 on $g_{M C L-1}$ & Fit \\
\hline $\mathrm{SBCL}-2_{\mathrm{B}}$ & 0.53 & - & Effect ST2 on $g_{B C L-2}$ & Fit \\
\hline SBIM & 0.49 & - & Effect ST2 on $g_{\text {BIM }}$ & Fit \\
\hline SNOXA & 0.40 & - & Effect ST2 on gNOXA & Fit \\
\hline$\gamma$ & 0.43 & $\mathrm{~d}^{-1}$ & Max. effect size BAX* ${ }^{*}$ & Fit \\
\hline$\kappa$ & 1.78 & - & Max. effect Caspase 12 & Fit \\
\hline$\beta$ & 2.65 & - & Inhibition strength ST2 on Caspase 3,7 & Fit \\
\hline $\mathrm{d}_{\mathrm{MCL}-1}$ & 16.4 & $\mathrm{~d}^{-1}$ & Decay rate MCL-1 & {$[30]$} \\
\hline $\mathrm{d}_{\mathrm{BCL}-2}$ & 0.86 & $\mathrm{~d}^{-1}$ & Decay rate BCL-2 & {$[30]$} \\
\hline $\mathrm{d}_{\mathrm{BIM}}$ & 5.94 & $\mathrm{~d}^{-1}$ & Decay rate BIM & {$[32]$} \\
\hline $\mathrm{d}_{\text {NOXA }}$ & 32.8 & $\mathrm{~d}^{-1}$ & Decay rate NOXA & {$[33]$} \\
\hline $\mathrm{d}_{\mathrm{BAX}}$ & 1.38 & $\mathrm{~d}^{-1}$ & Decay rate BAX & {$[31]$} \\
\hline $\mathrm{K}_{\mathrm{d}, 3}$ & 2.0 & $\mathrm{nM}$ & Dissociation constant & {$[24]$} \\
\hline $\mathrm{K}_{\mathrm{d}, 4}$ & 22.0 & $\mathrm{nM}$ & Dissociation constant & {$[24]$} \\
\hline $\mathrm{K}_{\mathrm{d}, 5}$ & 40.0 & $\mathrm{nM}$ & Dissociation constant & {$[24]$} \\
\hline $\mathrm{K}_{\mathrm{d}, 6}$ & 2.50 & $\mathrm{nM}$ & Dissociation constant & {$[24]$} \\
\hline $\mathrm{K}_{\mathrm{d}, 7}$ & 68.0 & $\mathrm{nM}$ & Dissociation constant & {$[24]$} \\
\hline $\mathrm{k}^{+}$ & 0.17 & $\mu \mathrm{M} \mathrm{d}^{-1}$ & Complex association rate & - \\
\hline $\mathrm{k}_{1}$ & 43.2 & $\mu \mathrm{M} \mathrm{d}^{-1}$ & BAX activation rate & - \\
\hline $\mathrm{k}_{2}$ & 8.64 & $\mu \mathrm{M} \mathrm{d}^{-1}$ & $\mathrm{BAX}^{*}$ deactivation rate & - \\
\hline $\mathrm{g}_{\mathrm{p}, 0}$ & 0.86 & $\mu \mathrm{M} \mathrm{d}^{-1}$ & Basal protein growth & - \\
\hline$\alpha$ & 10.0 & - & Inhibition strength APRIL on Caspase 12 & - \\
\hline $\mathrm{K}_{\mathrm{BAX}}$ & 200 & $\mathrm{nM}$ & Half-saturation constant BAX-halflife & - \\
\hline
\end{tabular}


Signal $\mathrm{S}_{1}$

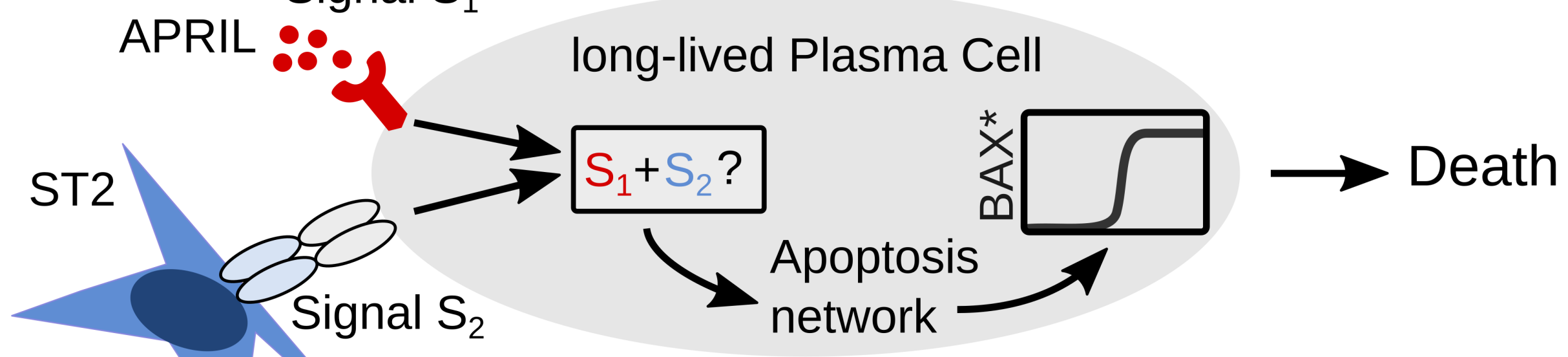

B

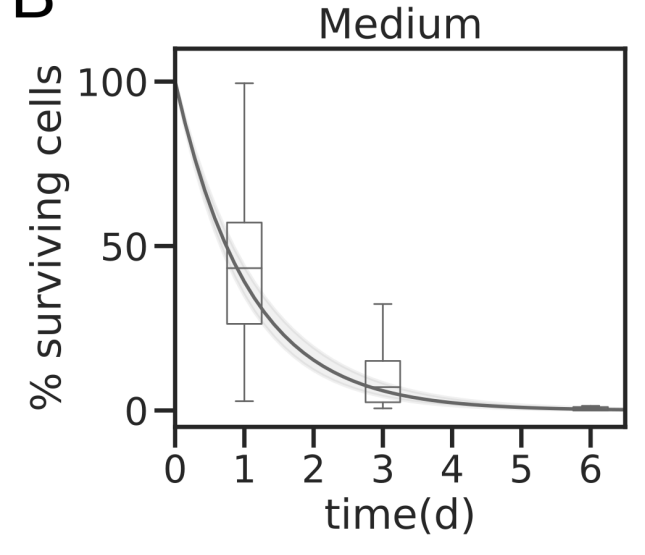

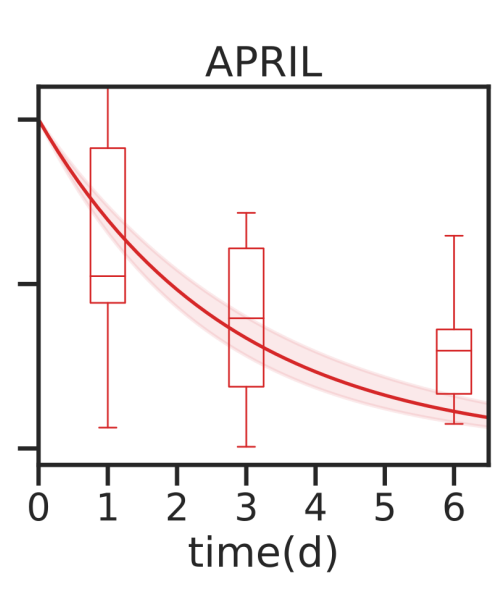

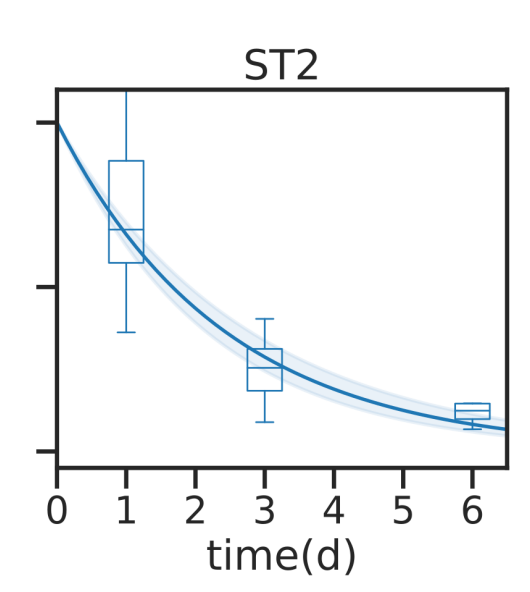

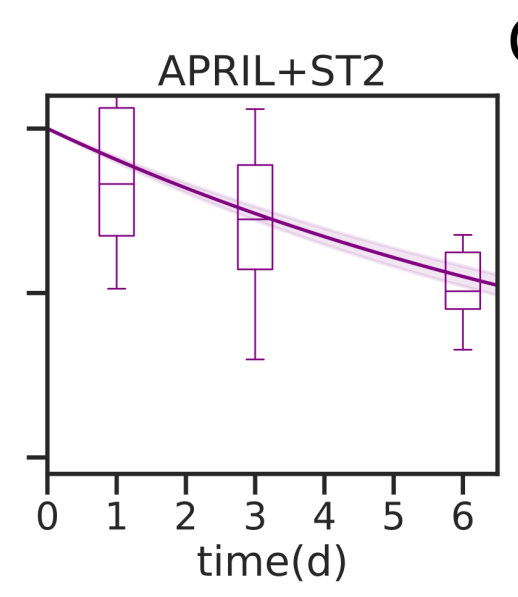

C

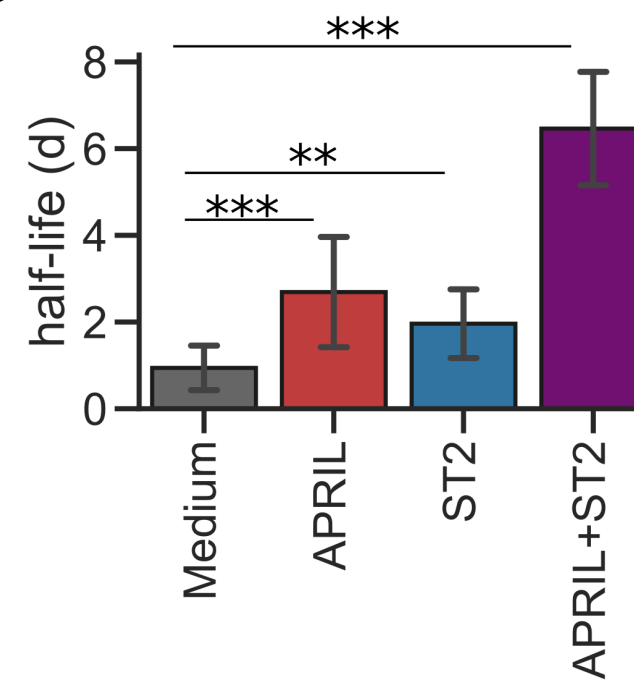

Burt et al. Figure 1 
A
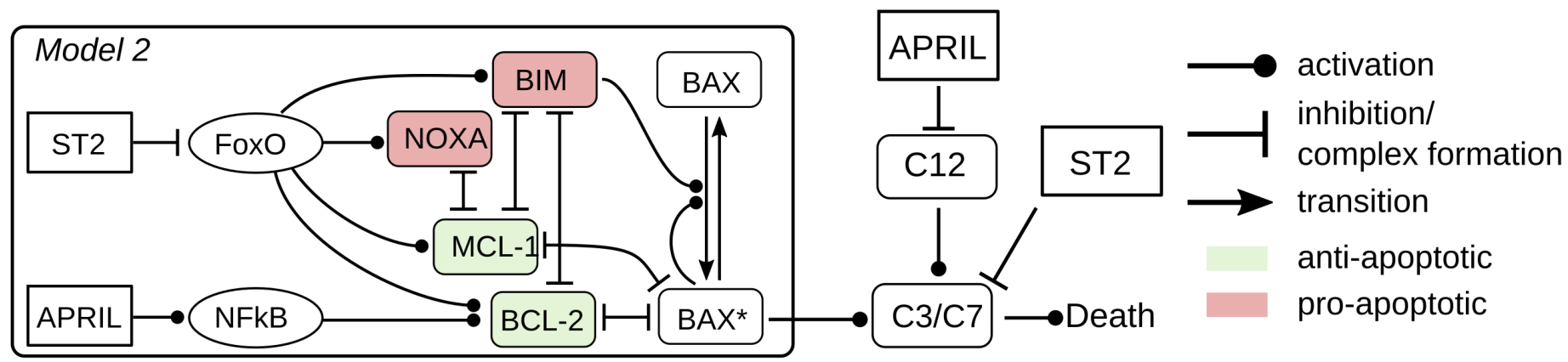

B

Data

Best Model fit
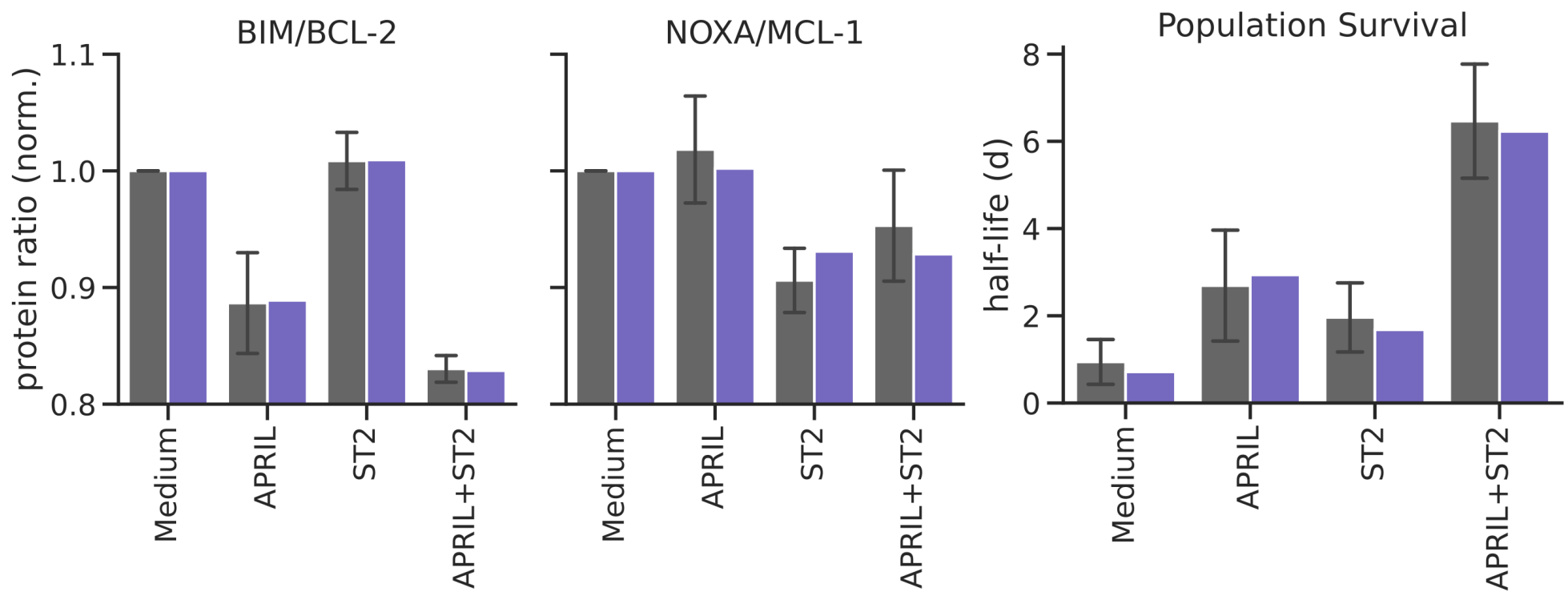

D
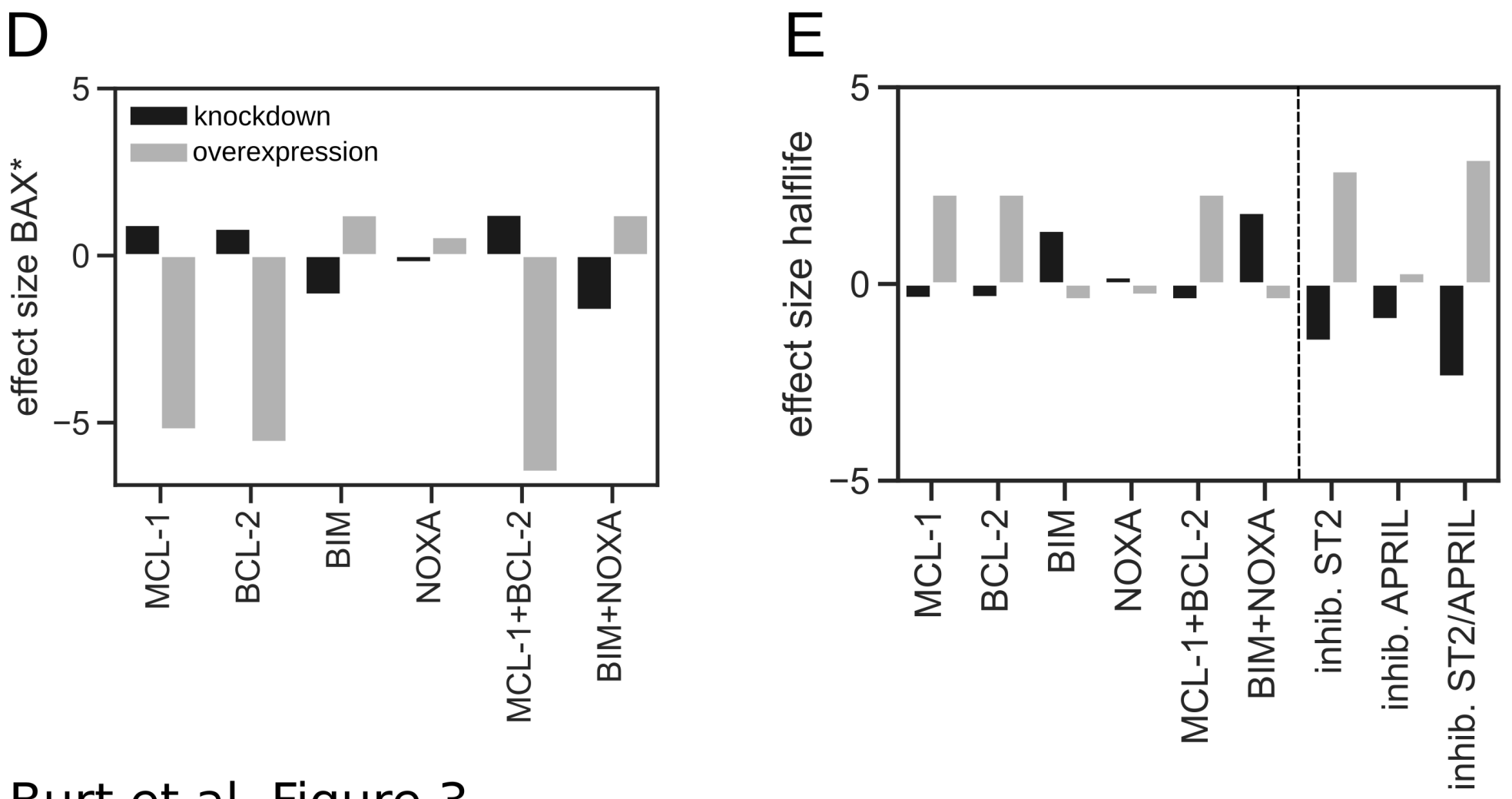

Burt et al. Figure 3 
